\title{
An integrated bioreactor-expanded bed adsorption system for the removal of acetate to enhance the production of alpha-interferon-2b by Escherichia coli
}

\begin{abstract}
A stirred tank bioreactor (STB) integrated with an expanded bed adsorption (EBA) system containing anion-exchange resin (Diaion WA30) was developed for in situ removal of acetate to increase the production of Ŭ-interferon-2b (Ŭ-PrIFN-2b) by Escherichia coli (E. coli). Although the total acetate $(9.79 \mathrm{~g} / \mathrm{L})$ secreted by E. coli in the integrated STB/EBA system was higher than that in a bioreactor with dispersed resin or a conventional batch bioreactor, cell growth $(14.97 \mathrm{~g} / \mathrm{L})$ and ŬPrIFN-2b production $(867.4 \mathrm{gg} / \mathrm{L})$ were significantly improved owing to the high efficiency of acetate removal from the culture. The production of ÜPrIFN$2 \mathrm{~b}$ in the integrated STB/EBA system was improved by 3 -fold and 1.4-fold over that obtained in a conventional batch bioreactor and a bioreactor containing dispersed resins, respectively.
\end{abstract}

Keyword: Bioreactors; Expanded bed adsorption; Fermentation; Acetic acid; Ŭ-Interferon-2b 